Abstracts on this page have been chosen and edited by Dr Trevor Watts

Geriatric dentistry; nutrition

\section{Oral status and nutrition in the institutionalized elderly \\ Lamy M, Mojon Ph et al. J Dent 1999; 27: 443-448}

Eating was a difficult undertaking for edentulous subjects with no dentures or only one.

In 8 nursing homes in the Liège vicinity, 120 subjects were examined with respect to oral condition, nutrition and contentment with oral condition. Subjects with age $\geq 65$ yrs, no cognitive impairment and no acute illness were selected from 276 residents, and divided into 3 groups: (1) 24 subjects edentulous without dentures or only one, (2) 43 with two complete dentures, (3) and 44 who were dentate with or without partial dentures.

Three quarters of subjects were female, and mean age was $81 \mathrm{yrs}$. All subjects had at least one medical problem, of which the most prevalent were cardiovascular diseases, depression and diabetes mellitus. About half were unable to perform a test of eating raw carrots.

Serum albumin did not differ between the three groups, but there was a difference between groups 1 and 2 in the mean Mini Nutritional Assessment (21.4 v. 23.6; group 322.3 ) which estimates size, general health, eating practices and self-perceived health. Significant differences included: the proportion who ate mashed food, which was twice as large in group 1; in this group, more reported difficulty in eating hard foods, and fewer found eating pleasant.

Oral habits and their association with signs and symptoms of temporomandibular disorders in adolescent girls

\section{Gavish A, Halachmi $M$ et al. J Oral Rehabil 2000; 27: 22-32}

Gum chewing, non-functional jaw movements and leaning the head on the arm were all related to increased signs and symptoms of temporomandibular disorders (TMD) in this study.

A random sample of 248 girls aged 15-16 from a school in central Israel, were examined for presence of TMD and occlusal signs, and completed a questionnaire on TMD related symptoms and oral habits. Gum was chewed by $92 \%$ of subjects for a mean reported time of $3.1 \mathrm{~h} / \mathrm{dy}, 76 \%$ chewed ice and icepops, and the most frequent reported symptoms were joint noises (44\%), joint pain (29\%) and joint catching $(21 \%)$, a sudden sticking of the jaw which is selfreleasing. Four girls reported joint locking.

Chewing gum for more than $3.1 \mathrm{~h} /$ dy was associated with joint noises and muscle sentivity on palpation. Observed jaw play unintended small mandibular movements without tooth contact - was associated with joint noises and catching. Leaning the head on the arm was associated with joint noises, catching and a feeling of tension within the joint. The authors suggest that patients with harmful parafunctional habits should be alerted to the possible problems which might occur.
Paediatric dentistry

Caries in five-year-old children and associations with family-related factors

\section{Mattila M-L, Rautava P et al. J Dent Res 2000; 79: 875-881}

Hygiene behaviour and age of parents, and cohabitation without marriage, are related to caries in children.

In the Finnish Family Competence Study, a sample of 1,582 women who visited maternity clinics for the first time in 1986 were approached, and 1,443 agreed to participate. When children reached the age of 5 yrs and there were 1,292 potential participants, 1,003 health questionnaires were returned. Dental examinations gave data for 828 of the children.

In 598 children, $\mathrm{dmft}$ was 0 , and this was associated with higher educational and occupational status of the mothers. Younger mothers more frequently had children with caries experience: $55 \%$ of children for mothers giving birth aged $<20$. Caries was also more frequent with younger fathers, fathers who had since remarried, and parents cohabiting without marriage; but it was less frequent in children of single mothers than those of married mothers.

Presence of caries related to included rural rather than urban living, and both parents' hygiene habits and caries experience. The authors conclude that caries control in children requires attention to the whole family.

\title{
Oral Health in America: A Report of the Surgeon General
}

\section{U.S. Department of Health and Human Services, National Institute of Dental and Craniofacial Research, National Institutes of Health. Rockville, MD, 2000.}

This report is an account of oral health in the US population, with summaries of research evidence.

The US Surgeon General publishes occasional reports on different aspects of health in the USA. Last year an excellent report was published on mental health, and now the spotlight has been turned on oral and dental health. The complete report is available at $h t t p: / / w w w . n i d c r . n i h . g o v / s g r / o r a l h e a l t h . h t m$ on the internet.

Apart from the emphasis on caries (the commonest chronic disease of childhood) and periodontal diseases, tumours and mucosal diseases and the relationship of oral and systemic health, there are interesting sections, for instance, on quality of life, cultural differences and the impact of oral conditions on communication. Researchers and clinicians will find much of interest.

Some important findings are demographic: cleft lip/palate affects whites 3 times as often as African Americans; children from poor families suffer 12 times as many days of restricted activity on account of oral diseases; 5 year oral cancer survival in whites is $56 \%$ and in blacks, $34 \%$. Although the report relates to the US scene, the listed evidence is pertinent to all developed countries. 